\title{
A PREDICTIVE TOXICITY STUDY OF PEIS, PAMAM AND ZAC DENDRIMERS
}

\author{
CLAUDIU N. LUNGU ${ }^{a^{*}}$, CSABA PAIZS ${ }^{a}$, MELINDA E. FÜSTÖS ${ }^{a}$, \\ ANAMARIA ORZA ${ }^{\mathrm{a}}$, MIRCEA V. DIUDEA $^{\mathrm{a}}$, \\ IRENEUSZ P. GRUDZINSKI ${ }^{\text {b }}$
}

\begin{abstract}
Polyethyleneimines (PEIs) and polyamidoamine dendrimers (PAMAM) are widely used as non-biological transporters in modern gene therapies. In this study, several zero-generation aromatic core "ZAC" dendrimers were computationally studied for predictive toxicity assessment. The chemical synthesis of the ZAC dendrimers was carried out using three different bromomethylbenzene derivatives as aromatic core and dialkanolamines as branching units with different carbon chain length. Quantitative structureactivity relationships (QSAR) and virtual high throughput toxicity screening (vHTTS) assays were applied on the synthesized zero generation dendrimers to evaluate their toxicities.
\end{abstract}

Keywords: molecular descriptors, pharmacophore, QSAR, continuous structures, toxicity, maximum recommended daily dose, dendrimers.

\section{INTRODUCTION}

Quantum structure activity relationship (QSAR) methods are widely used in toxicity prediction [1]. A QSAR model is used to predict accurately compounds properties that belong to the same chemical space as the compounds used in training set [2]. Small molecules or molecular fragments

a Department of Chemistry, Faculty of Chemistry and Chemical Engineering, Babeș-Bolyai University, Arany János Str 11, 400028, Cluj, Romania

b Department of Applied Toxicology, Faculty of Pharmacy, Medical University of Warsaw, Banacha 1, 02-097 Warsaw, Poland

*Corresponding author: lunguclaudiu5555@gmail.com 
are ideal tools of exploring a chemical space. In case of macromolecular compounds (e.g., polymers, dendrimers) building regression models based on parts of these molecules is not an accurate approach mainly because in the materials science parts of a structure don't preserve a proportional properties amount (in comparison to ligand-receptor systems, where the fragment-based concept is well known).

Testing toxicity especially in case of nanostructures is expensive mostly because toxicity properties are not additive. Being applied on a series of compounds (or molecular fragments) with similar chemical structure, these methods not always return correct data. Errors are due to both nonadditive properties and degeneration of variables (i.e., descriptors) used in building QSAR models. The back trucking may be avoided using virtual High Throughput Toxicity Screening (vHTTS) [3]. This method provides a large amount of unique/specific data for building a QSAR toxicity model.

Selecting small compounds with known toxicities, one covers a large region of the chemical toxicity space, making the model more powerful in terms of prediction.

\section{RESULTS AND DISCUSSIONS}

PAMAM and PEI properties were analyzed. Figure1 illustrates their QSAR property space [4]. Chemical space shapes are relatively the same for all PEI, PAMAM and ZAC dendrimers, respectively.

All PAMAM and PEls herein analyzed for genotoxic and carcinogenic properties do not have such effects due to computation studies. The computational studies for the Ames test (Salmonella typhimurium) also turned the negative results for all the compounds studied here. The in silico structure biodegrability test predicted a class 2 persistent chemical (tertiary amine) for all compounds. Computation tests performed for biotransformation processes due to cytochrome P450 evidenced for PAMAM both N-dealkylation and $\mathrm{N}$-oxidation, respectively. In vitro micronucleus assay based on QSAR showed a $\mathrm{H}$ acceptor path $3 \mathrm{~h}$ acceptor class 1 for PAMAM series. A multiple linear regression model was computed using PEI and PAMAM. The computed pharmacophores are shown in Figure 2. 

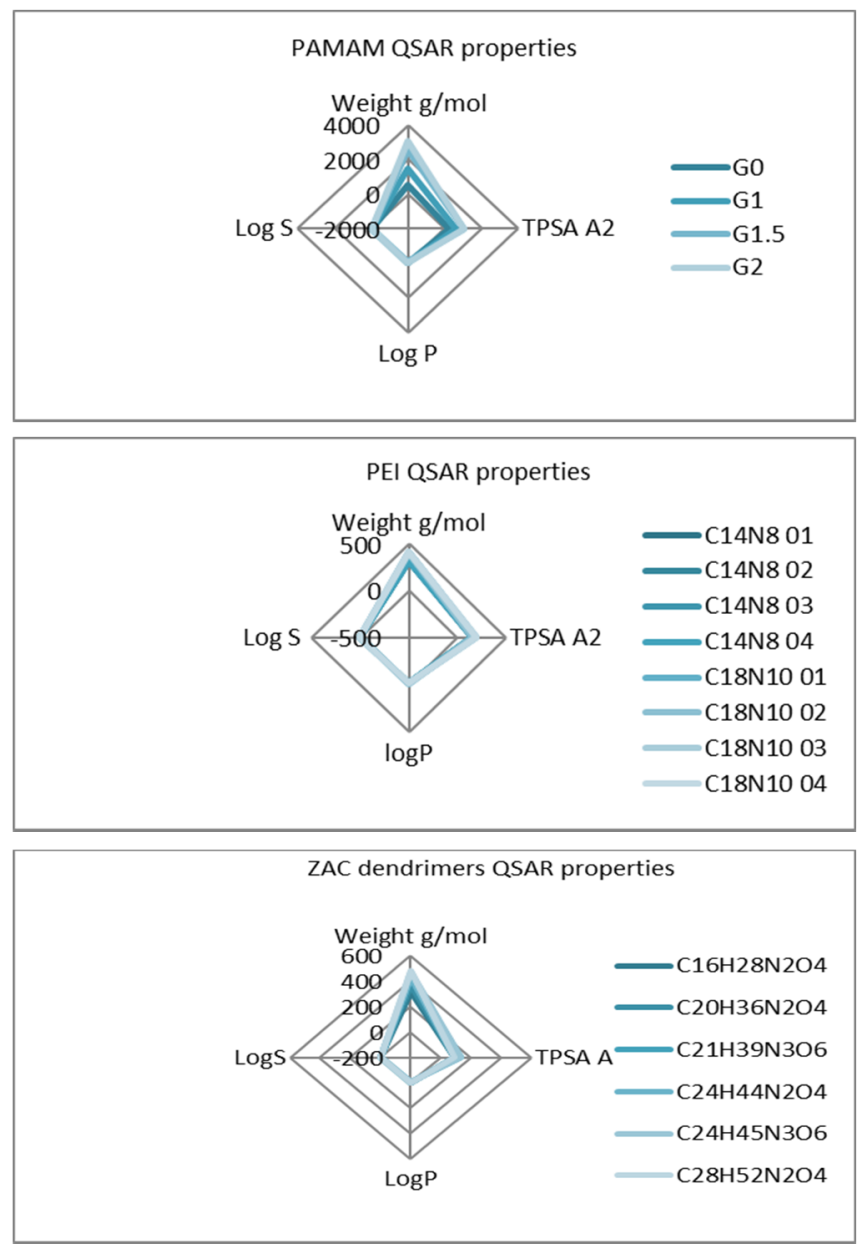

Figure 1. PAMAM/PEI and ZAC dendrimers QSAR properties

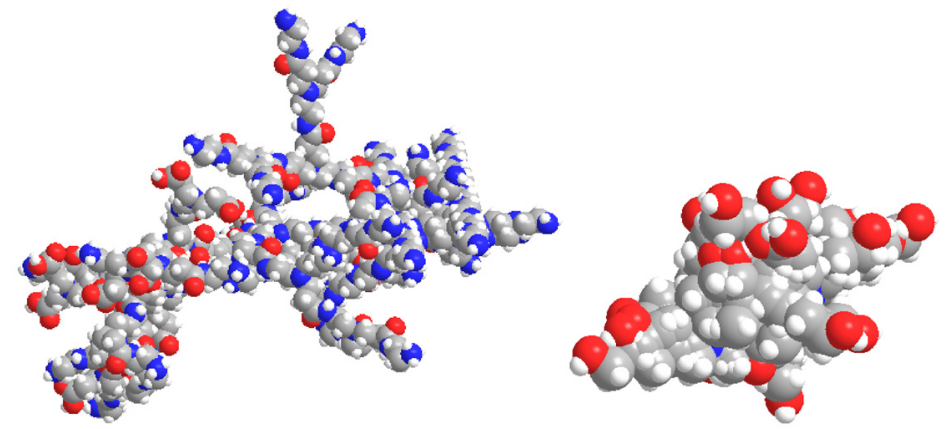

Figure 2. Pharmacophore structures form left to right: PEI/PAMAM and ZAC pharmacophore, respectively 
CLAUDIU N. LUNGU, CSABA PAIZS, MELINDA E. FÜSTÖS, ANAMARIA ORZA, MIRCEA V. DIUDEA, IRENEUSZ P. GRUDZINSKI

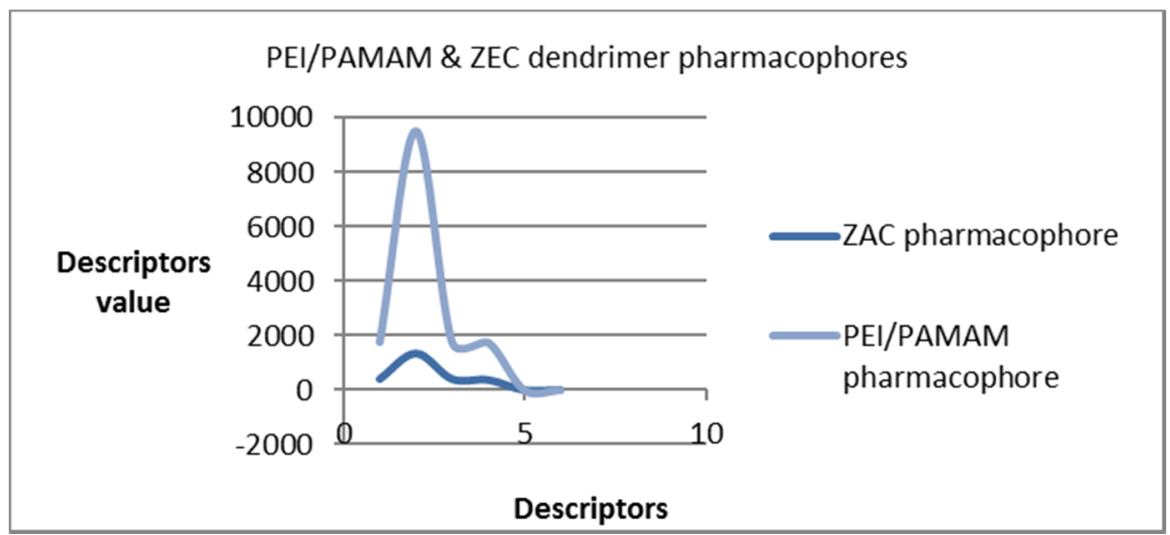

Figure 3. Topological properties of PEI/PAMAM and ZAC dendrimers. Topological properties are represented (from left to right) as follows: 1 cluster count; 2 polar surface area $A^{2}$ (PSA); 3 shape attribute; 4 sum of valence degrees; 5 total connectivity; 6total valence connectivity

After minimization of the two pharmacophores (for PEI/PAMAM and ZAC) and consecutively hydrogen bond forming, continuous structures resulted, as shown in Figure 4.
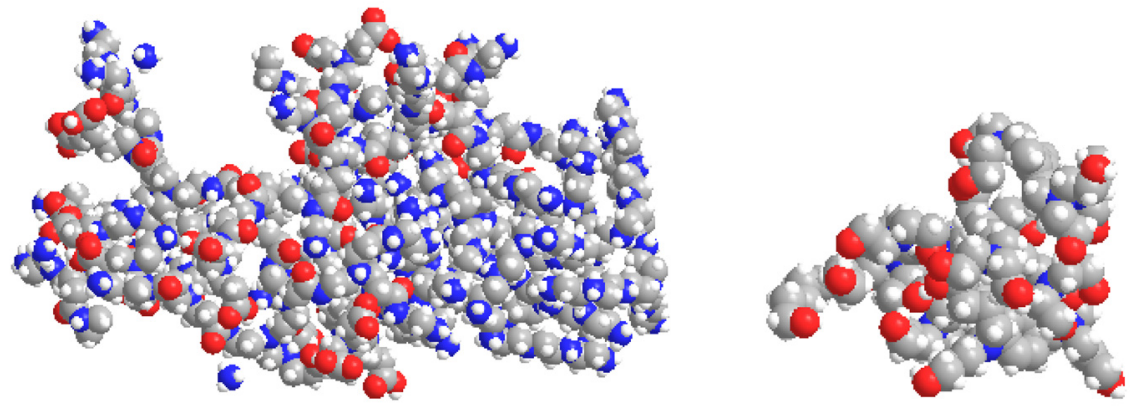

Figure 4. PEI/PAMAM and ZAC dendrimers pharmacophore molecules

Topological properties for the newly resulted molecules (derived from PEI/PAMAM and ZAC pharmacophores) and for the corresponding pharmacophores are shown in Figure 5. 


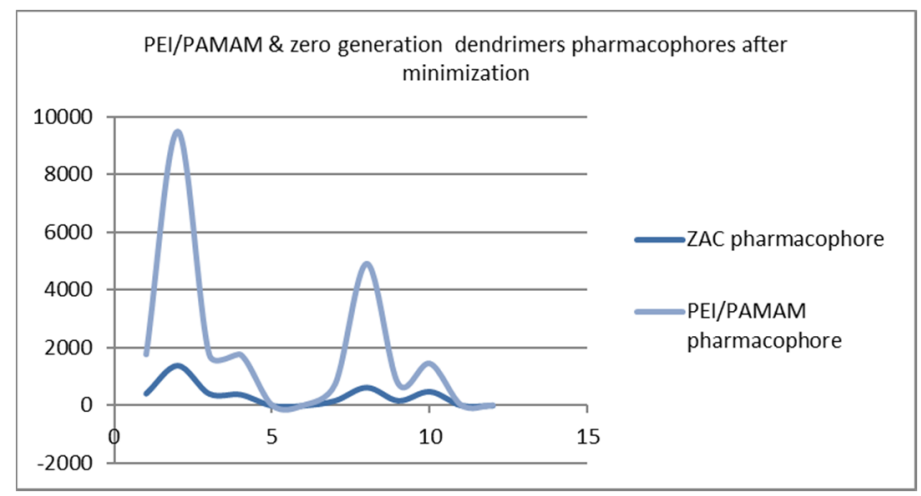

Figure 5. Topological properties of PEI/PAMAM and ZAC dendrimers, newly resulted molecules. Topological properties are represented (from left to right) as follows: 1 cluster count; 2 polar surface area $A^{2}(P S A) ; 3$ shape attribute; 4 sum of valence degrees; 5 total connectivity; 6total valence connectivity; 7 cluster count minimized; 8 polar surface area minimized $A^{2}(P S A) ; 9$ shape attribute minimized; 10 sum of valence degrees minimized; 11 total connectivity minimized; 12 total valence connectivity minimized.

Multiple linear regression model $[5,6,7]$ was obtained having as independent variable the acute toxicity and as dependent variables the number of $\mathrm{H}, \mathrm{C}, \mathrm{N}$ atoms, molecular weight, total number of atoms, number of heavy atoms, rotation degree, number of hydrogen accepting and donor groups, Anderson charges, minimum distance between two hydrogen distinct donor groups, minimum distance between an $\mathrm{H}$-donor and an $\mathrm{H}$-accepting group, mean distance between two $\mathrm{H}$-donor groups, mean distance between an $\mathrm{H}$-donor and an $\mathrm{H}$-accepting group, Wiener index, $\log \mathrm{P}$. All data were used on the training set, resulted in a Pearson correlation of $\mathrm{R}^{2}=0.996$ with $y=0.00165321+0.991084 x$; the cross validated square was 0.991084 (Figure 6).

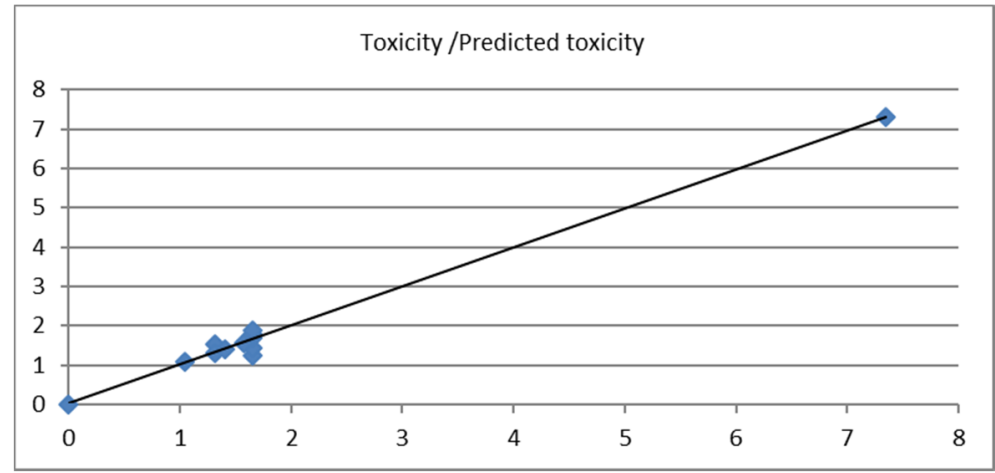

Figure 6. Correlation between toxicity doses used to model the data and the predicted toxicity doses 
CLAUDIU N. LUNGU, CSABA PAIZS, MELINDA E. FÜSTÖS, ANAMARIA ORZA, MIRCEA V. DIUDEA, IRENEUSZ P. GRUDZINSKI

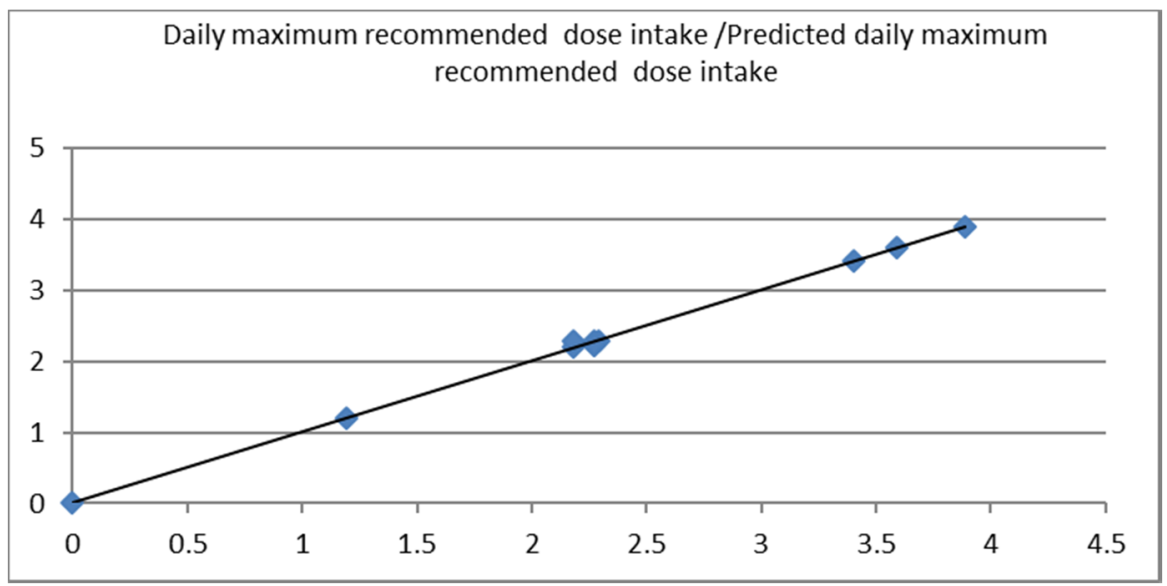

Figure 7. Correlation between daily maximum dose intake used to model data and predicted dose intake.

A model was computed for the prediction of daily dose intake using the maximum daily dose intake as the independent variable $[8,9,10]$; Person correlation value was $R^{2}=0.999$ with $y=0.00274498+0.998815 x$, with cross validated square of $Q^{2}=0.998815$ (Figure 7). The $K$ test was applied for the model in Figures 7-9 and no out layers were detected. Models were validated using leave one out technique.

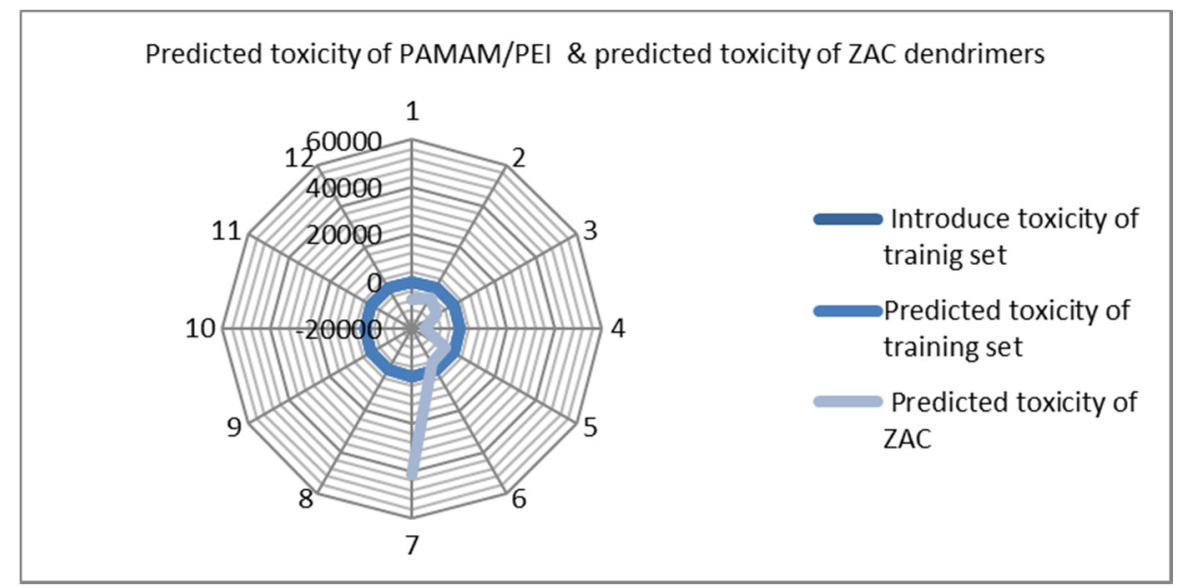

Figure 8. Toxicity values obtained for the studied series using the QSAR model. 


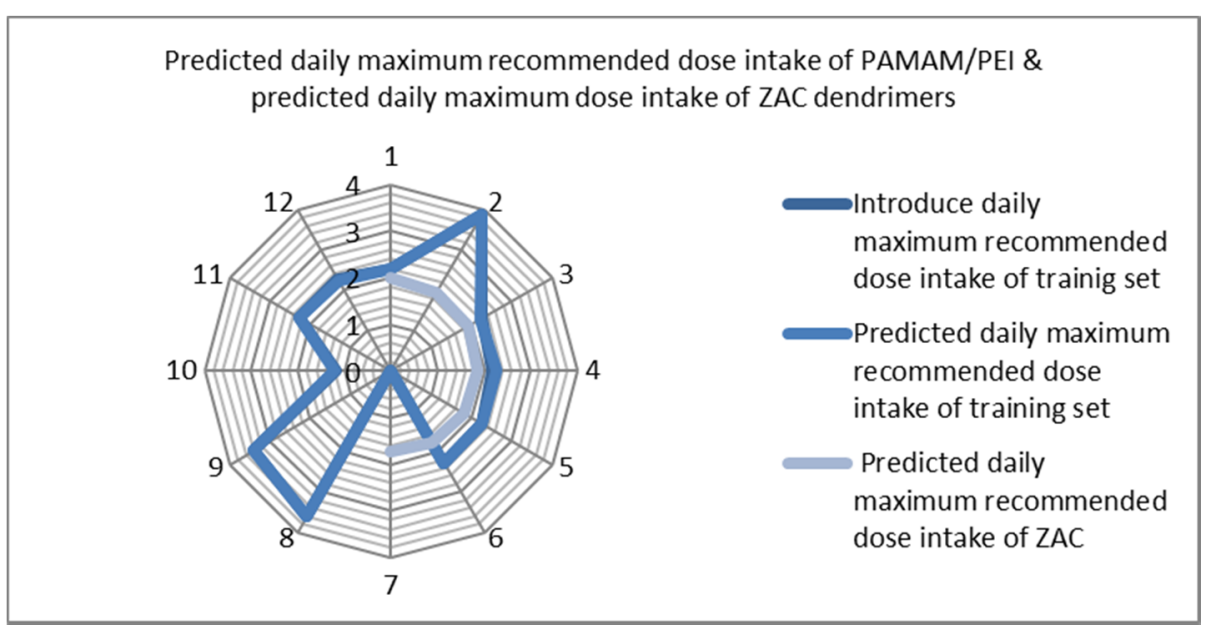

Figure 9. Daily maximum recommended doses for the dendrimer series using the QSAR model.

Data show that ZAC dendrimers are less recommended than the PAMAM/PEI compounds.

\section{CONCLUSIONS}

PEls and PAMAM dendrimers have no evidences on genotoxic effect based on computational models established for the AMES test. All these polymers are classified as the class 2 persistent chemical (tertiary amine). In silico tests for cytochrome P450-based biotransformation processes evidence for PAMAM some $\mathrm{N}$-dealkylation and $\mathrm{N}$-oxidation biotransformation pathways having possible interaction with drugs metabolized by the same CYP isoforms. In silico computational micronucleus assay has shown a $\mathrm{H}$-acceptor path $3 \mathrm{H}$-acceptor class 1 for PAMAM series expressing its potential interaction with the genetic material. The synthesized ZAC dendrimers are found to be 1000 times more toxic than PAMAM and PEls in terms of acute toxicity and maximum daily intake dose as predicted by the QSAR model, which showed a decrease in doses of the ZAC dendrimers comparative to PEI and PAMAM, respectively.

The chemical space of QSAR properties of the model and of the ZAC compounds (see Figure 1) have the same shape and thus the prediction of the model is presumably accurate. 
Furthermore, by computed topological properties of both pharmacophores, it was suggested that there is a slight overlap of the training (PAMAM/PEI) pharmacophore and the pharmacophore of ZAC dendrimers. By this reason, the correlation of these properties with the total connectivity and total valence connectivity is less predictive.

Having the same QSAR space, respectively the same topological variation of properties as the training (PAMAM/PEI) set and also high Pearson correlation $\left(R^{2}=0.996\right.$ and 0.999 , respectively) it is expected that ZAC dendrimers will have reduced doses than PEI/PAMAM (i.e., the acute dose for ZAC is 1000 smaller than for PEI/PAMAM).

\section{EXPERIMENTAL SECTION}

The studied ZAC dendrimers were chemically synthesized according to the literature procedure described by Füstös et al. [11]. To data, 1,4-bis(bromomethyl)benzene, 1,3,5-tris(bromomethyl) benzene and 1,3,5-tris(bromomethyl)-2,4,6-trimethylbenzene were used as the aromatic core. Derivatization was performed with the commercially available diethanolamine (DEA) and the "ad-hoc" prepared dipropanolamine, dibutanolamine and dipentanolamine, respectively. General procedure of preparation for ZAC compounds \# 1-6 is shown in Figure 10. To a solution of bromomethylbenzene in acetonitrile, the corresponding dialkanolamine was added under continuous stirring.

In order to avoid the dialkanolamine hydrobromide salt formation due to the resulting hydrobromic acid, potassium carbonate was added in excess. The reaction mixture was refluxed for 24 hours, being monitored on thin layer chromatography (eluent hexane:toluene 7:3). The resulted precipitate was separated by vacuum filtration, while the filtrate was evaporated at reduced pressure to give the crude product. After purification the dendrimers were obtained as viscous lightly yellowish liquids. Their structure was confirmed by NMR $\left({ }^{1} \mathrm{H},{ }^{13} \mathrm{C}\right)$ spectroscopy and mass spectrometry [12]. A polyamidoamine (PAMAM)/polyethylenimines (PEI) database was generated in order to perform a vHTTS.

A pharmacophore model (see below) was developed for the 6 ZAC compounds listed in Figure 10. A vHTTS screening was performed on this database (6 aromatic core dendrimers +8 PEI + 4 PAMAM structures) and the results were sent to the QSAR procedure (5). PAMAM (of generation 0; $1 ; 1.5$ and 2$)$ and PEI $\left(\mathrm{C}_{14} \mathrm{~N}_{8}\right.$ and $\left.\mathrm{C}_{18} \mathrm{~N}_{10}\right)$ were computed, in order to establish their chemical space and finally their own toxicity. The toxicity 
assessment due to QSAR model was computed using a decision tree software [13]. In order to establish the reliability of the model, pharmacophores used for generating the model (PEI/PAMAM pharmacophore) and the pharmacophore of ZAC dendrimers were studied topologically.
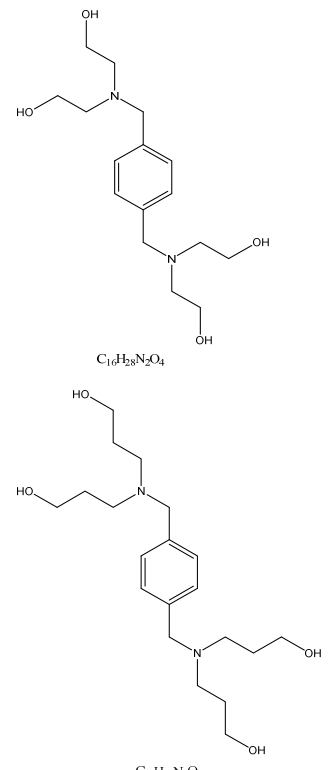

$\mathrm{C}_{20} \mathrm{H}_{36} \mathrm{~N}_{2} \mathrm{O}_{4}$
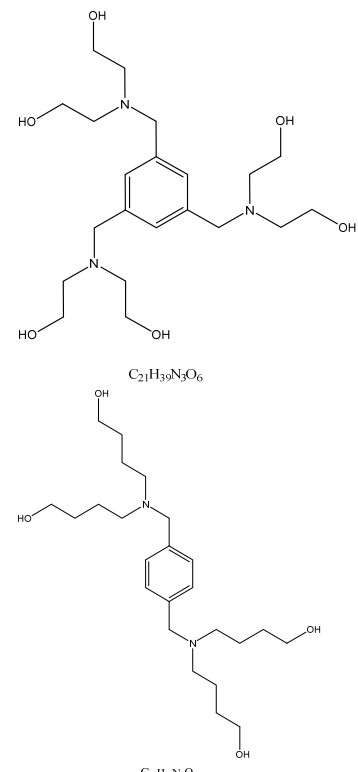

$\mathrm{C}_{2} \mathrm{H}_{4+4} \mathrm{~N}_{2} \mathrm{O}_{4}$
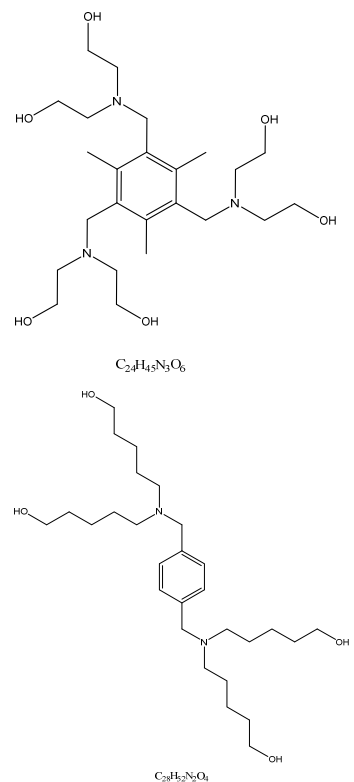

Figure 10. Synthesized ZAC dendrimers-target for vHTTS.

\section{ACKNOWLEDGMENTS}

This work was supported by GEMNS project granted in the European Union's Seventh Framework Program under ERA-NET EuroNanoMed II (European Innovative Research and Technological Development Projects in Nanomedicine).

\section{REFERENCES}

1. R. Hood, Developmental and reproductive toxicology: a practical approach, 3rd ed., CRC, Boca Raton, FL, 2011.

2. V.C. Arena; N.B. Sussman; S. Mazumdar; S. Yu; O.T. Macina; SAR QSAR Environ. Res., 2004,15, 1-18.

3. C. Hansch; A. Leo; S.B. Mekapati; A. Kurup; Biorg. Med. Chem., 2004, 12, 33913400 .

4. T. Langer; G. Wolber; Pure. App. Chem., 2004, 76, 991-996. 
CLAUDIU N. LUNGU, CSABA PAIZS, MELINDA E. FÜSTÖS, ANAMARIA ORZA, MIRCEA V. DIUDEA, IRENEUSZ P. GRUDZINSKI

5. M.E. Füstös; T.A. Sipos; M.V. Diudea; G. Katona; Croat. Chem. Acta, 2015, 88, 129-132.

6. M.V. Diudea; C.N. Lungu; C.L. Nagy; Molecules, 2018, 23, 2533.

7. C.N. Lungu; S. Ersali; B. Szefler; A. Pirvan-Moldovan; S. Basak; M.V. Diudea; Studia UBB, Chemia, 2017, 62, 197-204.

8. A. Vedani; M. Dobler; M. Smieško; Toxicol. Appl. Pharmacol., 2012, 261, 142-153.

9. A.J. Leo; C. Hansch; Perspect. Drug. Discov. Design, 1999,17, 1-25.

10. C.N. Lungu; M.V. Diudea; M.V. Putz; Int. J. Mol. Sci., 2017, 18, 1377.

11. S. Kim; E.E. Bolton; S.H. Bryant; J. Cheminform., 2011, 3, 26.

12. R. Perkins; H. Fang; W.D. Tong; W.J. Welsh; Environ. Toxicol. Chem., 2003, 22, 1666-1679.

13. C.N. Lungu; B.I. Bratanovici; M.M. Grigore; V. Antoci; I. Mangalagiu; Curr. Med. Chem., 2018, doi: 10.2174/0929867326666181220094229. 